\title{
Enhancements of Genetic Algorithm for Minimum Cost Design of Steel Frames Considering Fabrication Cost
}

\author{
Kiichiro Sawada ${ }^{1}$, Akira Matsuo ${ }^{2}$ and Hitoshi Shimizu ${ }^{3}$ \\ 1. Department of Architecture and Architectural Engineering, Kagoshima University, Kagoshima 890-0065, Japan \\ 2. Department of Architecture, Hiroshima University, Hiroshima 739-8527, Japan \\ 3. Hiroshima Branch Office, Takenaka Corporation, Hiroshima 730-0015, Japan
}

\begin{abstract}
A discrete optimization problem for minimizing the sum of fabrication cost and steel material cost of steel frames under constraints based on Japanese seismic code is set up. Enhancements of the genetic algorithm for the above-mentioned problem are proposed, which are combined with a 1D (one-dimensional) search or a 2D (two-dimensional) search. After the proposed methods are described, they are applied to a five-story frame. A comparison with an exact solution obtained by a revised enumeration algorithm demonstrates the effectiveness of the proposed methods.
\end{abstract}

Key words: Genetic algorithm, steel frames, fabrication cost.

\section{Introduction}

Numerous studies have been conducted on the structural optimization of steel frames. Many of these studies do not use an overall cost but use a steel structural weight as an objective function [1, 2], because the steel structural weight is approximately proportional to steel material cost. However, the overall cost includes not only steel material cost but also fabrication cost and erection cost. The steel structural weight is not necessarily proportional to the overall cost because fabrication cost depends on the complexity of the connections rather than the structural weight. A cost function considering the nodal cost for truss optimization [3] and some fabrication time functions [4, 5] have been proposed previously. Recently, a relatively simple fabrication time function for the rigid frames of steel buildings has been proposed [6].

Some researches $[5,7]$ use such cost functions for structural optimization. The advantages are as follows:

Corresponding author: Kiichiro Sawada, Dr., associate professor, research fields: structural optimization, corroded steel structures, and seismic design. E-mail: kich@aae.kagoshima-u.ac.jp. (a) Cost-saving is achieved by the minimum cost optimization [7]; and (b) The minimum cost solution is more adaptable for practical structural designs than the minimum weight solution [7].

On the other hand, the difficulties are that mathematical programming methods cannot be used and even nature-inspired and biology-inspired algorithms such as the GA (genetic algorithm) suffer from redundancy in convergence and a dependency on random seeds because of remarkable discontinuity of fabrication cost function.

In this study, a discrete optimization problem for minimizing the sum of fabrication cost and steel material cost of steel frames under constraints based on the Japanese seismic code is set up. Enhancements of the genetic algorithm for the above-mentioned problem are proposed, which are combined with a 1D (one-dimensional) search or a 2D (two-dimensional) search. After the proposed methods are described, they are applied to a five-story frame. A comparison with an exact solution obtained by a revised enumeration algorithm demonstrates the effectiveness of the proposed methods. 


\section{Minimum Cost Design Problem of Steel} Frames

\subsection{Typical H-Beam-RHS-Column Connection in Japan}

Fig. 1a shows a typical H-beam-RHS-column connection in Japan. At the steel fabricating company, the box column connection is first welded to two through diaphragms using full-penetration welds, as shown in Fig. 1b. The connection is welded to the flanges of the bracket by full-penetration welds and to the web of the bracket by fillet welds as shown in Fig. 1c. Finally, the columns are welded to the connection by full-penetration welds as shown in Fig. 1d. In the field, the bracket is connected to the beam by high strength bolts. This study deals with buildings having beam-to-column connections shown in Fig. 1.

\subsection{Steel Fabrication Time Function [6]}

In this study, the following function is used for predicting the steel fabrication time:

$$
T F=T P+T B+T W+T I
$$

where, $T F$ represents the steel fabrication time, $T P$, the preparatory process time, $T B$, the assembly time, $T W$, the welding time, and $T I$, the time for preparing shop drawings.

The preparatory process consists of the marking and drilling of diaphragms, the marking, drilling and blasting of brackets and the marking, drilling, blasting and flanging bevels of beams. Responses to questionnaires sent to three steel fabricating companies in the Chugoku area, Japan [6], indicated that the preparatory process time depends on the number of parts such as diaphragms, beams and brackets rather than the structural weight. The following function is proposed to estimate the preparatory process time $T P$.

$$
T P=K P \cdot\left(\sum_{i=1}^{n j} N P_{D_{i}}+\alpha_{P B} \cdot N P_{B}\right)
$$

where, $N P_{D i}$ represents the number of diaphragms for the beam-to-column connection $I, n j$, the number of beam-to-column connections, and, $N P_{B}$, the number of beams and brackets, $\alpha_{P B}$ and $K P$ represent the coefficients for evaluating the preparatory process time.

The responses to the questionnaires [6] indicated that the assembly time depends on the number of elements rather than the structural weight. Therefore, the function $T B$ for estimating the assembly time is expressed as follows:

$$
T B=K B \cdot\left(\sum_{\mathrm{i}=1}^{n j} N B_{0_{i}}+\alpha_{B C} \cdot N B_{C}\right)
$$

where, $N B_{0 i}$ represents the number of elements consisting of connection panels and brackets for the beam to column connection $i, N B_{C}$ represents the number of columns and $\alpha_{B C}$ and $K B$ represent the coefficients for evaluating the assembly time.

Furthermore, the responses [6] showed that the welding time depends on the sum of the jointed sectional areas. The following function is proposed to estimate the welding time $T W$.

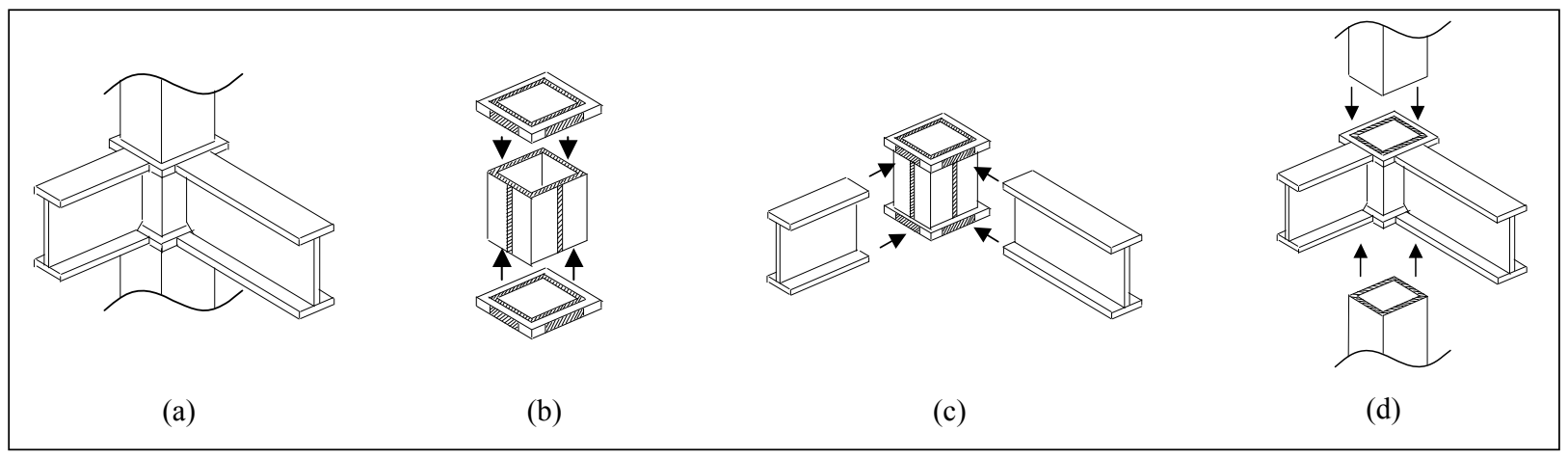

Fig. 1 Typical beam-to-column connection in Japan. 


$$
T W=K W \cdot\left(\sum_{i=1}^{n j} A_{D i}+\sum_{i=1}^{n b b} A_{B B_{i}}\right)
$$

where, $A_{D i}$ represents the jointed sectional area between the column and the diaphragm for the beam to column connection $i, A_{B B i}$ represents the jointed sectional area between the column and the bracket, $n b b$ represents the number of brackets and $K W$ represents the coefficient for evaluating the welding time.

Since the time for preparing shop drawings depends on the number of sheets of shop drawings, the following function, based on the number of columns and beams, is proposed:

$$
T I=K I c \cdot N I c+K I b \cdot N I b+K I g \cdot W
$$

where, NIC represents the number of shop fabricated column trees, NIb represents the number of beam groups having the same cross-sectional size, $W$ represents the total structural weight of the frame and $K I c, K I b$ and $K I g$ represent the coefficients used for evaluating the time for preparing the shop drawings.

\subsection{Coefficients to Evaluate the Fabrication Time [6]}

The values of $\alpha_{P B}$ and $\alpha_{B C}$ in Eqs. (2) and (3) were calculated from the responses of the questionnaires on the fabrication time as follows.

$$
\alpha_{P B}=2, \alpha_{B C}=7
$$

On the basis of the recorded fabrication time data of only one fabricating company, the values of $K P, K B$, $K W, K I b, K I c$ and $K I g$ in Eqs. (2), (3), (4) and (5) were computed from the least squares approximation as follows: $K P=0.85(\mathrm{~h}), K B=1.07(\mathrm{~h}), K W=0.013$ $\left(\mathrm{h} / \mathrm{cm}^{2}\right), K I b=2.87(\mathrm{~h}), K I c=4.12(\mathrm{~h}), K I g=0.067$ $(\mathrm{h} / \mathrm{kN})$

2.4 Computational Examples of $N P_{D i}$ in Eq. (2), $N B_{0 i}$ in Eq.(3) and $A_{D i}$ in Eq.(4) for Beam to Column Connections

The values of $N P_{D i}$ in Eq. (2), $N B_{0 i}$ in Eq. (3) and $A_{D i}$ in Eq. (4) for beam-to-column connections are computed as illustrated by the following examples.

For a standard beam-to-column connection shown in Fig. $2 \mathrm{a}, N P_{D i}=2, N B_{0 i}=7$, and $A_{D i}=4 \mathrm{Ac}_{\mathrm{i}}$, where $A c_{i}$ is the cross sectional area of the column adjoining connection $i$.

For a beam-to-column connection including an internal diaphragm, as shown in Fig. $2 \mathrm{~b}, N P_{D i}=3, N B_{0 i}$ $=8$, and $A_{D i}=5 A c_{i}$.

For a beam-to-column connection having different upper and lower column depths shown in Fig. 2c, $N P_{D i}$ $=2, N B_{0 i}=10$, and $A_{D i}=4 A c_{i}+4 H b_{i} \cdot T c_{i}$, where $H b_{i}$ is the cross sectional depth of the beam adjoining connection $i$ and $T c_{i}$ is the thickness of the lower column adjoining connection $i$.

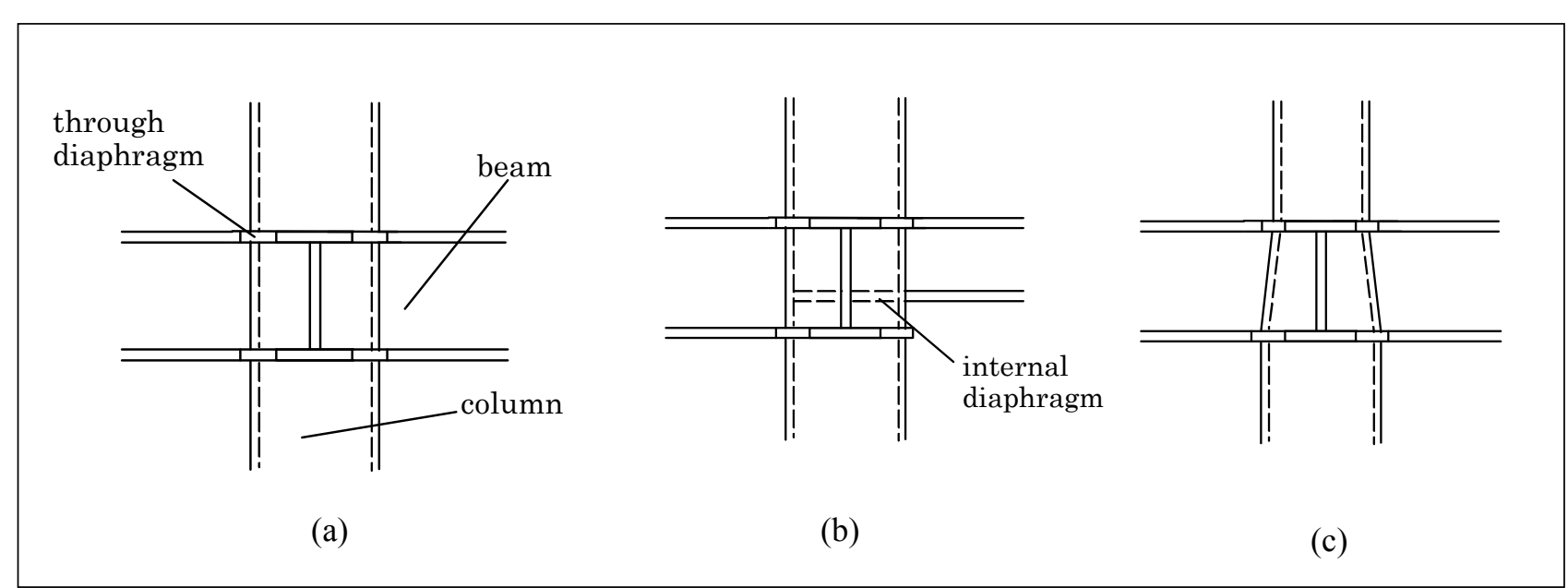

Fig. 2 Various beam-to-column connections. 


\subsection{Minimum Cost Design Problem}

The minimum cost design problem considered in this study is described below.

$$
\text { Find } D_{i d c}(i d c=1, \ldots, N D C), H_{i d b}(i d b=1, \ldots, N D B)
$$

which minimize

$$
F=K F \cdot T F+K S \cdot\left(\rho \sum_{i=1}^{N M} A_{i} L_{i}+\rho \sum_{i=1}^{N J} V_{D i}\right)
$$

subjected to

$$
\begin{aligned}
& g_{L i}=\frac{N_{L i}}{A_{i} f_{N L i}}+\frac{M_{L i}}{Z_{i} f_{M L i}} \leq 1 \quad(i=1,2, \ldots, N M) \\
& g_{S i}=\frac{N_{S i}}{A_{i} f_{N S i}}+\frac{M_{S i}}{Z_{i} f_{M S i}} \leq 1 \quad(i=1,2 \ldots, N M) \\
& g_{D k}=\frac{\delta_{k} / H_{k}}{1 / 200} \leq 1 \quad(k=1,2 \ldots, N F) \\
& g_{C B R k}=\sum_{i} M_{P C k i} / \sum_{i} 1.5 M_{P B k i} \geq 1(k=1, \ldots, N F) \\
& g_{P}=\lambda_{P} \geq 1
\end{aligned}
$$

where, $D_{i d c}$ is the index number of the standard section of the steel box column and $H_{i d b}$ is the index number of the standard section of the steel H-section. In Eq. (6), $F$ denotes the total cost of the steel framework fabrication and steel material (hereafter referred to as the total cost). The variables $N D C, N D B$, $N M$ and $N J$ denote the total number of column design variables, total number of beam design variables, total number of members, and total number of joints, respectively. $T F$ refers to the steel fabrication time function given in Eq. (1), and $K F$ and $K S$ denote the fabrication labor cost per hour and the steel material unit cost, respectively. $\rho, A_{i}$ and $L_{i}$ are the specific gravity of steel, member cross-sectional area, and member length, respectively. $V_{D i}$ is the diaphragm volume of the $i$-th joint. The constraints are in accordance with the Building Standard Law of Japan [8]. In Eqs. (7a-7e), $g_{L i}$ denotes the constraint function when loaded vertically, $g_{S i}$ denotes the constraint function when simultaneously loaded both vertically and horizontally, $g_{D k}$ represents the constraint function for the story drift angle, $g_{C B R k}$ denotes the constraint function for the beam-to-column strength ratio, and $g_{P}$ indicates the constraint function for the collapse load of the framework. $N_{i}, M_{i}, f_{N i}, f_{M i}$ and $Z_{i}$ denote the axial load, bending moment, allowable stress under the axial load and bending, and section modulus for each member, respectively. Furthermore, the variables $\delta_{k}, H_{k}$, and $N F$ represents the story drift, story height, and the number of stories, respectively. The variables $M_{P C K i}$ and $M_{P B K i}$ denote the strengths of the column and beam members, respectively, and $\lambda_{p}$ represents the load factor with respect to the design horizontal loads for the formation of the collapse mechanism.

\section{Genetic Algorithm}

Although an enumeration algorithm [9] or a branch and bound method [10] is valuable as an exact method, a considerably large amount of computational effort is required. Recently, nature-inspired and biologyinspired algorithms (such as the GA (genetic algorithm), SA (simulated annealing), the ACO (ant colony optimization) algorithm and the PSO (particle swarm optimization) algorithm have been applied to various problems. Although most of these algorithms cannot assure the achievement of global optima, they can seemingly obtain the global or near-global optima with less computational effort than the exact branch and bound method or the enumeration. Numerous studies have applied a GA to discrete truss problems. The generation of a good initial population [11], with a combination of a GA with stress heuristics [12] and local search [13] has been proposed to reduce the computational effort. However, in discontinuous cost function optimization such as that mentioned in this study, GA-based methods seem to suffer from redundancy in convergence and dependency on random seeds. Here, a combination of the GA with a simple 1D (one-dimensional) search technique, which selects a member section randomly as a search axis, or a 2D (two-dimensional) search technique, which selects some pairs of member sections effect on the cost function as search axes, is proposed.

\subsection{Real Coding, Crossover and Mutation}

Real coding [14] has recently been investigated for 
continuous optimization in order to achieve high precision. In this study, real coding is adopted not for continuous but for discrete problems for an easy application of the 1D or the 2D search technique. The advantages of real coding for the discrete problem are as follows: (1) the number of genes for an individual is considerably small, (2) the conversion of genotype to phenotype is relatively simple, (3) the range of a parameter does not have to be a power of two, and (4) 1D or 2D search can be combined with a GA without difficulty. Fig. 3 shows an example of real coded genes and the conversion of genotype into discrete phenotype. A string consisting of random numbers from 0 to 1 is used as a genotype. In this study, a single-point crossover and uniform mutation [15] are used. The proposed algorithm has an additional routine, the simple 1D search or 2D search, as shown in Fig. 4.

\subsection{Simple One-Dimensional Search}

The first technique is a simple 1D search [16, 17]. Before crossover, some individuals are selected randomly, and the design variable axes for the individuals are selected randomly as the search directions. Next, gene $R_{s, i}$ is changed temporarily one by one at a certain interval. If the reduction in the fitness function from the original value is confirmed, then gene $R_{s, i}$ is fixed. The procedure is described as follows:

Before crossover, the following steps are executed for each individual.

[Step0] Set $R_{S, 1}^{\prime}=R_{S, 1}, R_{S, 2}^{\prime}=R_{S, 2}, \ldots, R_{S, N D}^{\prime}=$ $R_{S, N D}$ for individual $\mathrm{s}$ ( $\left.\mathrm{s}=1,2, \ldots, \mathrm{NS}\right)$, where NS represents number of individuals and ND represents number of design variables (member sections).

[Step1] Set $i=0$

[Step2] $i=i+1$ : If $i$ is greater than $N D$ then go to the next individual.

[Step3] Go to Step4 with probability $q$. Go to Step2 with probability 1- $q$.

[Step4] Set $j 1=0$

[Step5] $j 1=j 1+1$ : If $j 1$ is greater than $N$ then go to
Genotype information for individual s

$\mathrm{R}_{\mathrm{S} 1}=0.69 \quad \mathrm{R}_{\mathrm{S} 2}=0.34 \quad \mathrm{R}_{\mathrm{S} 3}=0.89$

Conversion of genotype into phenotype

$$
\begin{aligned}
& 0.5 \leq R_{S 1}<0.75 \rightarrow \mathrm{A} 1=30 \mathrm{~cm}^{2} \\
& 0.25 \leq R_{S 2}<0.50 \rightarrow \mathrm{A} 2=10 \mathrm{~cm}^{2} \\
& 0.75 \leq R_{S 3} \leq 1.0 \rightarrow \mathrm{A} 3=40 \mathrm{~cm}^{2} \\
& \mathrm{~A} 1=30 \mathrm{~cm}^{2} \\
& \begin{array}{|c|c|}
\hline \text { ID. } & \text { Cross sectional area } \\
\hline 1 & 10.0 \mathrm{~cm}^{2} \\
\hline 2 & 20.0 \mathrm{~cm}^{2} \\
\hline 3 & 30.0 \mathrm{~cm}^{2} \\
\hline 4 & 40.0 \mathrm{~cm}^{2} \\
\hline
\end{array}
\end{aligned}
$$

Fig 3 An example of converting genotype into phenotype.

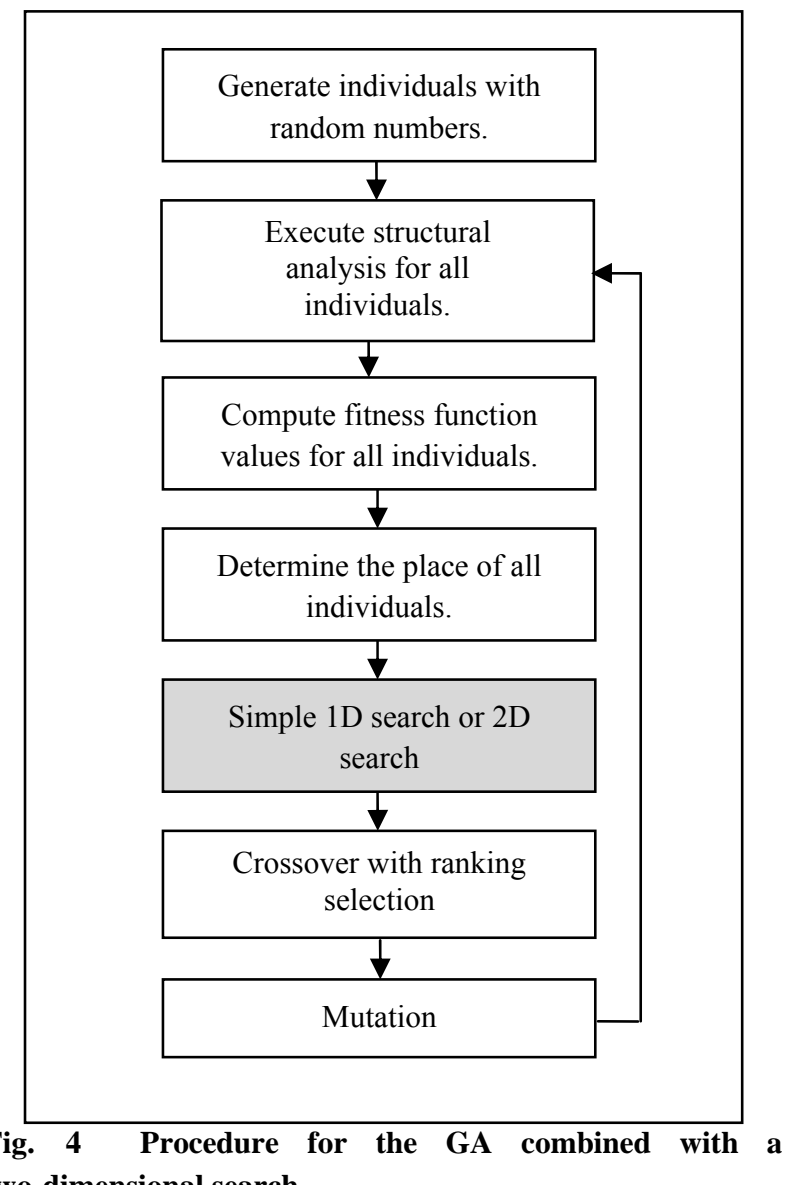
two-dimensional search.

Step2. ( $N$ represents the number of standard sections.) 
[Step6] Set $R_{s, I}^{\prime}=(j 1-0.5) / N$ to change a section of member $i$ to standard section $j l$ for the individual.

[Step7] Calculate the cost $F$ of the individual with genes $R_{s, i}^{\prime}$.

[Step8] If the following equation is satisfied, then go to Step9, else go to Step5.

$$
\gamma \cdot F_{o p c} \leq F \leq F_{o p c}
$$

$F_{\text {opc }}$ : Cost of the current optimum solution.

[Step9] Execute the structural analysis for the individual with genes $R_{s, i}$.

[Step10] Calculate the fitness function value for the individual with genes $R_{s, i}$.

[Step11] If the fitness function value is revised, set $R_{s, i}=R_{s, i}^{\prime}$ to update the discrete sections of individuals.

[Step12] Go to Step5.

\subsection{Two-Dimensional Search}

In the minimum cost design it is important to consider whether it is good to equalize the left and right beam depths (upper and lower column depths) at each connection. Before crossover, some individuals are selected randomly, and a given pair of the design variable axes for the individual is selected as the search directions. Next, the genes are temporarily changed one by one at a certain interval. If the reduction in the fitness function from the original value is confirmed, then the genes are fixed. The 2D search is executed as follows:

First, the following step is executed:

[Step0a] Input some pairs of member number $\left(J_{1}, K_{1}\right),\left(J_{2}, K_{2}\right), \ldots,\left(J_{i}, K_{i}\right), \ldots\left(J_{N P}, K_{N P}\right) \quad$ as the preassigned parameters.

Before crossover, the following steps are executed for all individuals.

[Step0b] Set $R_{S, 1}^{\prime}=R_{S, 1} R_{S, 2}^{\prime}=R_{S, 2}, \ldots, R_{S, N D}^{\prime}=$ $R_{S, N D}$ for individual $s$ ( $\left.s=1,2, \ldots, \mathrm{NS}\right)$, where $N S$ represents number of individuals and $N D$ represents number of design variables (member sections).

[Step1] Set $I=0$

[Step2] $i=i+1$ : If $i$ is greater than $N P$ then go to the next individual.
[Step3] Go to Step4 with probability $p$. Go to Step2 with probability 1-p.

[Step4] Set $j 1=0$ and $j 2=0$

[Step5] $j 1=j 1+1$ : If $j 1$ is greater than $N$ then go to Step2. ( $N$ represents the number of standard sections.)

[Step6] $k 1=k 1+1$ : If $k 1$ is greater than $N$ then go to Step5.

[Step7] Set $R_{S, J i}^{\prime}=(j 1-0.5) / N$ and $R_{S, K i}^{\prime}=$ $(k 1-0.5) / N$ to change the section of member $J_{i}$ to standard section $j l$ and the section of member $K_{i}$ to standard section $k l$ for the individual.

[Step8] Calculate the total cost $F$ of the individual with genes $R^{\prime}{ }_{S, J i}$ and $R^{\prime}{ }_{S, k i}$.

[Step9] If Eq. (8) is satisfied, then go to Step10, else go to Step6.

[Step10] Execute the structural analysis for the individual with genes $R_{S, J i}$ and $R_{S, k i}^{\prime}$.

[Step11] Calculate the fitness function value for the individual with genes $R_{S, J i}^{\prime}$ and $R_{S, k i}^{\prime}$.

[Step12] If the fitness function value is revised, set $R_{S, J i}=R_{S, J i}^{\prime}$ and $R_{S, K i}=R_{S, K i}^{\prime}$ to update the discrete sections of individual s.

[Step13] Go to Step6.

\section{Numerical Example}

\subsection{Five Story Three-Span Plane Frame}

Fig. 5 shows a 5 story 3 span plane frame (hereafter referred to as the 5 story frame). The number of design variables is 12: a column for each bottom layer (1, 2 stories) and top layer (3, 4 and 5 stories), external beam, and internal beam for each story. The vertical load is $6.72 \mathrm{kN} / \mathrm{m}^{2}$, which is the sum of the dead load and the live load. For elastic constraints, the horizontal loads are shown in Fig. 5, the horizontal loads (See Fig. 5) based on the Ai distribution of the shear forces story of the stories are used with the standard story shear force coefficient $\mathrm{C} 0[7]=0.2$. For plastic constraints, the horizontal forces based on Ds [7] $=0.4$, that is, two times of the horizontal forces shown in Fig. 5, are used. The available standard sections are listed in Tables 1 and 2. The column members are rectangular steel tubes 


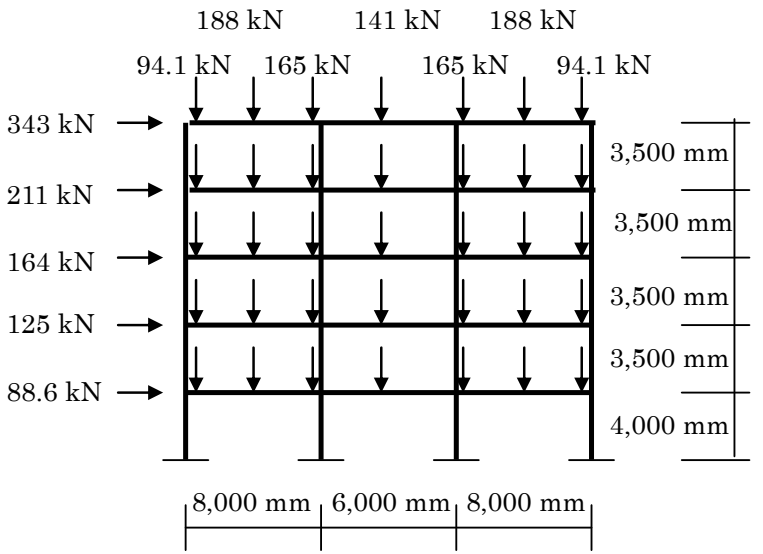

Fig. 5 Five-story, 3-span steel frame.

Table 1 Standard sections of columns (BCR295).

\begin{tabular}{lll}
\hline Series of columns & $\begin{array}{l}\text { ID of standard } \\
\text { sections }\end{array}$ & Standard sections \\
\hline \multirow{4}{*}{$\square 350$} & 1 & $\square 350 \times 12$ \\
& 2 & $\square 350 \times 16$ \\
& 3 & $\square 350 \times 19$ \\
& 4 & $\square 350 \times 22$ \\
$\square 400$ & 5 & $\square 400 \times 16$ \\
& 6 & $\square 400 \times 19$ \\
$\square 450$ & 7 & $\square 400 \times 22$ \\
& 8 & $\square 450 \times 16$ \\
$\square 500$ & 9 & $\square 450 \times 19$ \\
& 10 & $\square 450 \times 22$ \\
& 11 & $\square 500 \times 19$ \\
& 12 & $\square 500 \times 22$ \\
\hline
\end{tabular}

Table 2 Standard sections of beams (SN400).

\begin{tabular}{lll}
\hline Series of columns & $\begin{array}{l}\text { ID of standard } \\
\text { sections }\end{array}$ & Standard sections \\
\hline H400 & 1 & $\mathrm{H} 400 \times 200 \times 8 \times 13$ \\
H500 & 2 & $\mathrm{H} 500 \times 200 \times 10 \times 16$ \\
& 3 & $\mathrm{H} 500 \times 300 \times 11 \times 18$ \\
H600 & 4 & $\mathrm{H} 600 \times 200 \times 11 \times 17$ \\
H700 & 5 & $\mathrm{H} 600 \times 300 \times 12 \times 20$ \\
\hline$-D \times$ & 6 & $\mathrm{H} 700 \times 300 \times 13 \times 24$ \\
\hline
\end{tabular}

$\square-\mathrm{D} \times \mathrm{T}$ : Rectangular hollow section with depth $\mathrm{D}$ and thickness $\mathrm{T}$.

$\mathrm{H}-\mathrm{D} \times \mathrm{B} \times \mathrm{Tw} \times \mathrm{Tf}$ : H-section with depth $\mathrm{D}$, width $\mathrm{B}$, web thickness Tw and flange thickness Tf.

(BCR295), and the beam members are steel $\mathrm{H}$-sections (SN400). Young's modulus E is $2.06 \times 10^{5}\left(\mathrm{~N} / \mathrm{mm}^{2}\right)$. The unit cost of the steel material, KS, is 4,079 yen $/ \mathrm{kN}$ ( 40,000 yen/ton) and the steel fabrication labor cost per hour is $2,250 \mathrm{yen} / \mathrm{h}$.
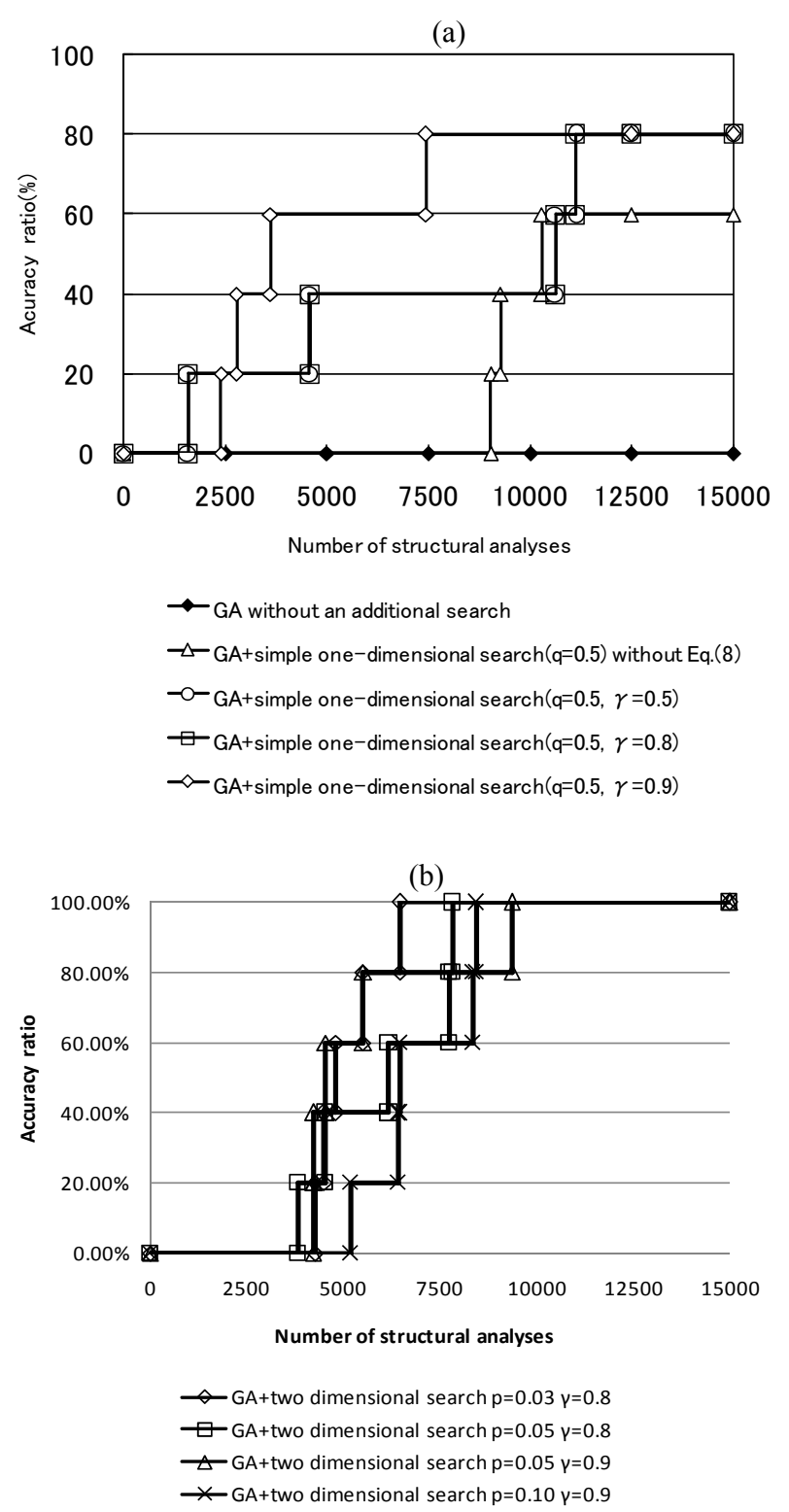

Fig. 6 elationship between the accuracy ratio and the number of structural analyses: (a) History of accuracy ratio (genetic algorithm with simple 1D search); (b) History of accuracy ratio (genetic algorithm with 2D search).

\subsection{Numerical Results}

Six pairs of members are used as the search axes in the $2 \mathrm{D}$ search, these are the columns of the bottom layer and the top layer, the external beam and the internal beam in each story. Fig. 6 shows the relationship between the accuracy ratio and the number of structural analyses. Here, the accuracy ratio represents the ratio of the number of runs that found a solution equal to the exact cost (Table 3 ) among five 
Table 3 Exact discrete solution with a revised enumeration [9].

\begin{tabular}{lllll}
\hline Member & Story & Sections & $\mathrm{A}\left(\mathrm{cm}^{2}\right)$ & $\mathrm{I}\left(\mathrm{cm}^{4}\right)$ \\
\hline \multirow{2}{*}{ Columns } & 3rd-5th & $\square 400 \times 16$ & 237.0 & 57,100 \\
& 1st-2rd & $\square 400 \times 19$ & 277.2 & 65,400 \\
Outside & 5th & $\mathrm{H} 500 \times 200 \times 10 \times 16$ & 112.2 & 46,800 \\
span & 4th & $\mathrm{H} 500 \times 200 \times 10 \times 16$ & 112.2 & 46,800 \\
beams & 3rd & $\mathrm{H} 600 \times 200 \times 11 \times 17$ & 131.7 & 75,600 \\
& 2nd & $\mathrm{H} 600 \times 200 \times 11 \times 17$ & 131.7 & 75,600 \\
& 1st & $\mathrm{H} 600 \times 300 \times 12 \times 20$ & 187.2 & 114,000 \\
Inside & 5th & $\mathrm{H} 500 \times 200 \times 10 \times 16$ & 112.2 & 46,800 \\
span & 4th & $\mathrm{H} 500 \times 200 \times 10 \times 16$ & 112.2 & 46,800 \\
beams & 3rd & $\mathrm{H} 600 \times 200 \times 11 \times 17$ & 131.7 & 75,600 \\
& 2nd & $\mathrm{H} 600 \times 300 \times 12 \times 20$ & 187.2 & 114,000 \\
& 1st & $\mathrm{H} 600 \times 200 \times 11 \times 17$ & 131.7 & 75,600 \\
Structural weight & \multicolumn{3}{c}{$254.97(\mathrm{kN})$} \\
Structural cost & $3,132,483(\mathrm{yen})$ \\
\hline
\end{tabular}

A: Cross sectional area, I: Moment of inertia

runs. The exact cost is obtained by the revised enumeration [9]. According to Fig. 6a, the proposed 1D search gives a better accuracy ratio and convergence than a case without an additional search. Fig. 6b shows that 2D searches provide a stable enhancement of the accuracy ratio and convergence over various parameters.

\section{Conclusions}

In this study, a discrete optimization problem for minimizing the sum of fabrication cost and steel material cost of steel frames under constraints based on Japanese seismic code was set up. Enhancements of the GA for the minimum cost problem were proposed, which were combined with $1 \mathrm{D}$ or $2 \mathrm{D}$ search. These methods were applied to a five-story frame. A comparison with the exact solution obtained by the revised enumeration algorithm revealed the effectiveness of the proposed methods.

\section{Acknowledgments}

This research was carried out with financial support from the Ministry of Education, Culture, Sports, Science, and Technology, Japan. We express our sincere gratitude for this assistance.

\section{References}

[1] K. Uetani, M. Tsuji and I. Takewaki, Application of an optimum design method to practical building frames with viscous dampers and hysteretic dampers, Engineering Structures 25 (5) (2003) 579-592.

[2] K. Sawada and A. Matsuo, An exact algorithm and approximate algorithms for discrete optimization of steel building frames, in: The third China-Japan-Korea Joint Symposium on Optimization of Structural and Mechanical Systems, 2004, pp. 663-668.

[3] M. Ohsaki, Genetic algorithm for topology optimization of trusses, Computers \& Structures 57 (2) (1995) 219-225.

[4] K. Jarmai and J. Farkas, Cost calculation and optimisation of welded steel structures, Journal of Constructional Steel Research 50 (1999) 115-135.

[5] L. Pavlovcic, A. Krajnc and D. Beg, Cost function analysis in the structural optimization of steel frames, Struct Multidisc Optim 28 (2004) 286-295.

[6] T. Sasaki, H. Shimizu, K. Sawada, A. Matsuo and A. Namba, A study on an estimation of steel fabrication cost based on the recorded fabrication times data, Journal of Constructional Steel 15 (2007) 79-84. (in Japanese)

[7] H. Shimizu, K. Sawada and A. Matsuo, Minimum cost design solution of optimization program in steel framework design for buildings [CD-ROM], in: IABSE-IASS Symposium, London, Sep. 20-23, 2011.

[8] The Building Center of Japan, The Building Standard Law of Japan, Japan, May, 2009.

[9] K. Sawada, A. Matsuo and H. Shimizu, Enhancements of discrete optimization algorithms for minimum cost design of steel frames considering fabrication cost [CD-ROM], in: 9th World Congress on Structural and Multidisciplinary Optimization, Shizuoka, Japan, June 13-17, 2011.

[10] M. Stolpe, Global optimization of minimum weight truss topology problems with stress, displacement, and local buckling constraints using branch-and-bound, International Journal for Numerical Methods in Engineering 61 (2004) 1270-1309.

[11] P. Ponterosso and D. S. J. Fox, Heuristically seeded genetic algorithm applied to truss optimization, Engineering with Computers 15 (1999) 345-355.

[12] W. Gutkowski, Z. Iwanow and J. Bauer, Controlled mutation in evolutionary structural optimization, Struct. Multidisc. Optim. 21 (2001) 355-360.

[13] T. Y. Chen and C. J. Chen, Improvements of simple genetic algorithm in structural design, International Journal for Numerical Methods in Engineering 40 (1997) 1323-1334.

[14] C. Z. Janikow and Z. Michalewicz, An experimental 
comparison of binary and floating point representation in genetic algorithms, in: R. Belew and L.B. Booker (Eds.), in: Proceedings of the Fourth International Conference on Genetic Algorithms, 1991, pp. 31-36.

[15] Z. Michalewicz and C. Z. Janikow, Handling constraints in genetic algorithms, in: R. Belew and L.B. Booker (Eds.), in: Proceedings of the Fourth International Conference on Genetic Algorithms, 1991, pp. 151-157.

[16] K. Sawada, H. Shimizu and A. Matsuo, Enhanced genetic algorithm with randomized line search techniques for discrete truss optimization [CD-ROM], in: 8th World Congress on Structural and Multidisciplinary Optimization, Lisbon, Portugal, June, 2009.

[17] K. Sawada, A. Matsuo and H. Shimizu, Randomized line search techniques in combined GA for discrete sizing optimization of truss structures, Struct Multidisc Optim. 44 (3) (2011) 337-350. 